\title{
Information load, group organization, and communication frequency*
}

\section{SIEGFRIED STREUFERT, THOMAS CAFFERTY, and FRANCES CHERRY Purdue University, Lafayette, Ind. 47907}

Four-man teams participated in a complex decision-making task. They were separated into decision-making and information acquisition dyads who could communicate via telephone only. All information was received by acquisition dyads who were free to call decision makers whenever they chose. The effect of suboptimal, optimal (one item of information per $3 \mathrm{~min}$ ), and superoptimal information load presented to acquisition dyads on their frequency of communications to decision makers was investigated. Increasing information load produced increasing communication frequency. The slope of the curve relating information load to communication frequency was linear but less slanted than a 1:1 input/output relationship would predict. Communication frequency was significantly different from, but more similar to, optimal information load than to experimentally induced information load. It appears that information acquisition groups can be of some value in improving information flow to decision makers. However, "imaginary" information may be transmitted to the decision makers when the amount of information received is low and suboptimal.

Complexity theory (Driver \& Streufert, 1966, 1969; Schroder, Driver, \& Streufert, 1967; Streufert \& Fromkin, in press) distinguishes between environmental effects on simple (e.g., respondent) and complex (e.g., integrated strategic) behavior. The theory proposes that optimal complex perceptual and behavioral processes should occur at intermediate information levels, i.e., when the person's or group's environment is neither overly rich in information input nor overly poor. Research by Streufert (1970), Streufert \& Driver (1965), and Streufert \& Schroder (1965) has repeatedly demonstrated that the most complex (most highly integrated) perceptual and performance indices of individual and group behavior are obtained when a person or group is exposed to one item of information every $3 \mathrm{~min}$, and that the quality of complex perception and performance decreases when information is received more or less frequently. Later research reported by Streufert, Driver, \& Haun (1967) has shown that this optimal effect holds specifically for complex perceptions and behavior (e.g., the processes involved in strategic decision making). Both individuals and groups appear quite able to process much more information into appropriate behavior if the responses are to be simple (e.g., respondent or retaliatory -in other words, when there is no need for the use of complex strategy. In many cases where complex behavior is attempted

* This research was supported by a contract between the Office of Naval Research, Group Psychology Branch, and Purdue University. or desirable, however, information is received less often or more often than once every $3 \mathrm{~min}$

Individuals or groups that are faced with the task of making strategic decisions in complex environments are often overloaded with information that reduces their capacity to operate in a complex strategic fashion. In other words, they are receiving more information than they can process optimally. A frequently employed solution for this problem is hiring a staff of personnel which is instructed to gather and sort information into categories for easier comprehension or to discard "unnecessary" information (e.g., information that is either irrelevant or marginal to the task at hand). Such information acquisition (gathering-sorting) staffs are frequently employed by high-level political and executive personnel Writers in social psychology and in human engineering have designated persons or groups fulfilling these functions as "human communication links" (cf. Campbell, 1958). It has been demonstrated repeatedly that the use of humans as part of an information transmission system tends to abbreviate, simplify, and condense incoming information, even when no instructions to that effect have been Bartlett, 1932; Campbell \& Gruen, 1958). According to Campbell (1958), this reduction of incoming information would be due to both random (variable) and constant (evolutionary adaptive) errors.

One might question whether errors, particularly constant errors in human information transmission between information gathering-sorting staffs given (e.g., Allport \& Postman, 1947 ; and decision-making staffs, should be classified as errors in the usual sense, or whether these errors function as adaptive aids in the decision-making process. The reduction of information transmitted by an information gathering-sorting staff to a decision-making staff might be of considerable value if (1) the quantity of information received by an information gathering staff was superoptimal and (2) the decision makers had to make complex (strategic) decisions.

Further, one might question whether the experimentally obtained reduction by human information transmission links is indeed ubiquitous (cf. Campbell, 1958). It may well be that the loss of information and the decreased transmission frequency are a function of the kind and quantity of information that Es have employed. Would similar losses in information be expected when little information or primarily irrelevant information was available, e.g., when information load is suboptimal (i.e., less than one item of information every $3 \mathrm{~min})$ ? Even under a suboptimal information load, some loss of information and communication frequency might be expected if human transmission links show the same characteristics under all conditions. However, some amount of increase in information and/or communication frequency should be expected if human transmission links function in an adaptive fashion.

The research reported in this paper is an attempt to investigate the effects of suboptimal, optimal, and superoptimal information loads (as repeatedly determined by complexity researchers) to which an information gathering-sorting staff is exposed on information transmission characteristics between the sorting staff and a decision-making staff.

If the information gathering-sorting staff operates as a simple link in the transmission system, then any increase (or decrease) in the amount of information received should produce an equivalent increase (or decrease) in the frequency of initiated information transmissions (potentially with lowered frequency of transmissions because of constant and variable transmission errors). On the other hand, the information gathering-sorting staff may operate adaptively to maintain an optimal level of information input to the decision makers (i.e., a level of approximately one item of information per $3 \cdot \mathrm{min}$ period $^{1}$ ). This "adaptive" operation would serve to increase information input to decision makers when available information is suboptimal and to decrease information when information levels are superoptimal 
Table 1

Mean Number of Telephone Calls Per Message Input Level

Level of Message Input

\begin{tabular}{cccccc}
2 & 6 & 10 & 14 & 18 & 22 \\
\hline 5.24 & 6.71 & 9.24 & 10.18 & 11.12 & 12.88 \\
\hline
\end{tabular}

(cf. Streufert, 1970; Streufert \& Driver, 1965; Streufert, Driver, \& Haun, 1967; Streufert \& Schroder, 1965). Assuming no instructions to operate in either fashion, what transmission characteristics would be obtained?

\section{METHOD}

Sixty-eight paid undergraduate volunteers from a large midwestern university participated in the tactical and negotiations game (TNG), a complex decision-making environment developed by Streufert and others (Streufert, Castore, \& Kliger, 1967; Streufert, Kliger, Castore, \& Driver 1967). In the TNG, Ss are given responsibility to make decisions about military, economic, intelligence, and diplomatic affairs in a simulated small-scale internation conflict with some Vietnam characteristics. Seventeen four-man teams were formed. The teams spent $2 \mathrm{~h}$ reading a manual about the game. The purpose of the manual is twofold: (1) to expose Ss to the complexities of the conflict with which they later have to deal, and (2) to provide $2 \mathrm{~h}$ of constant preexperimental exposure for all Ss in an attempt to somewhat equalize preexperimental experiences. After the reading period, Ss were instructed that they would be separated into two two-man groups. One group was to function as decision makers, and the other was to function as an information acquisition group (information gatherers and sorters). After a $10-\mathrm{min}$ period in which the four-man team was allowed to discuss its strategy, the teams were then separated and each of the two-man groups was placed in identical rooms, with a telephone as the only means of communication between groups. Information acquisition groups were instructed to pass "important" information to decision makers via the telephone. They were permitted to make as many calls as they wanted. However, the length of calls was limited to $1 \mathrm{~min}$ each (to avoid maintaining a continuous open line between the two groups). Ss were permitted, however, to make "call backs" if their communication had been interrupted by an automatic cancellation after a 1-min conversation. Calls were to be limited to the discussion of a single topic, e.g., a single piece of information. Calls were allowed in both directions: information acquisition groups were able to call their decision-making group, and vice versa. The maximum number of calls possible, if Ss intended to maintain continuous contact with each other, was 28. ${ }^{2}$ The maximum number of calls made by any team during any one period was 32 (less than $1 \mathrm{~min}$ per call). In other words, Ss' attempts to contact each other were not limited by the "technical" aspects of design or equipment.

Ss believed that they wre playing the TNG against another four-man team. In reality, information received by each team was preprogrammed. All information received by a team was submitted to the team's information acquisition group via a mail slot. Decision-making groups did not receive information from the Es; they were entirely dependent on information via telephone messages from their information acquisition groups. Information acquisition groups were not able to make decisions but were free to make "suggestions" to the decision makers. The decision-making group of each team made written decisions which were transmitted to the Es via a mail slot.

\section{DATA COLLECTION}

Teams of $\mathrm{Ss}$ participated in seven 30-min "playing periods." During the first (warm-up) period, the information acquisition group of each team received 10 written messages containing programmed information. (This period, in other words, presented teams with the optimal information load of one item of information every
3 min.) The teams were then exposed to six additional $1 / 2-h$ periods, during which data were collected. Each period exposed the information asquisition group of each team to an information load of $2,6,10,14,18$, or 22 written messages. The order of these information loads was randomized for each team.

The number of calls initiated by the information acquisition group of each team during each playing period was counted. Call backs were not included in the count.

\section{RESULTS}

Table 1 presents the mean number of calls initiated by the information acquisition group of each team at each level of information load. A single-factor repeated-measures ANOVA was performed on the data. The effect for load conditions was significant $(\mathrm{F}=45.99, \mathrm{df}=5 / 80$, $\mathrm{p}<.001)$. A subsequent Newman-Keuls analysis indicated significant $(\mathrm{p}<.05)$ differences between all pairs of load levels, except Levels 10 and 14 and Levels 14 and 18. Finally, a trend analysis on the data indicated a significant linear trend $(\mathrm{F}=224.85, \mathrm{df}=1 / 80, \mathrm{p}<.001)$ with no evidence for higher-order trends $(\mathrm{F}<1.00)$. Approximately $99 \%$ of the variance due to load conditions was accounted for by a linear function.

The data are plotted in Fig. 1. Each data point is accompanied by its appropriate $95 \%$ confidence interval to allow comparison against some alternative models. The lines of two

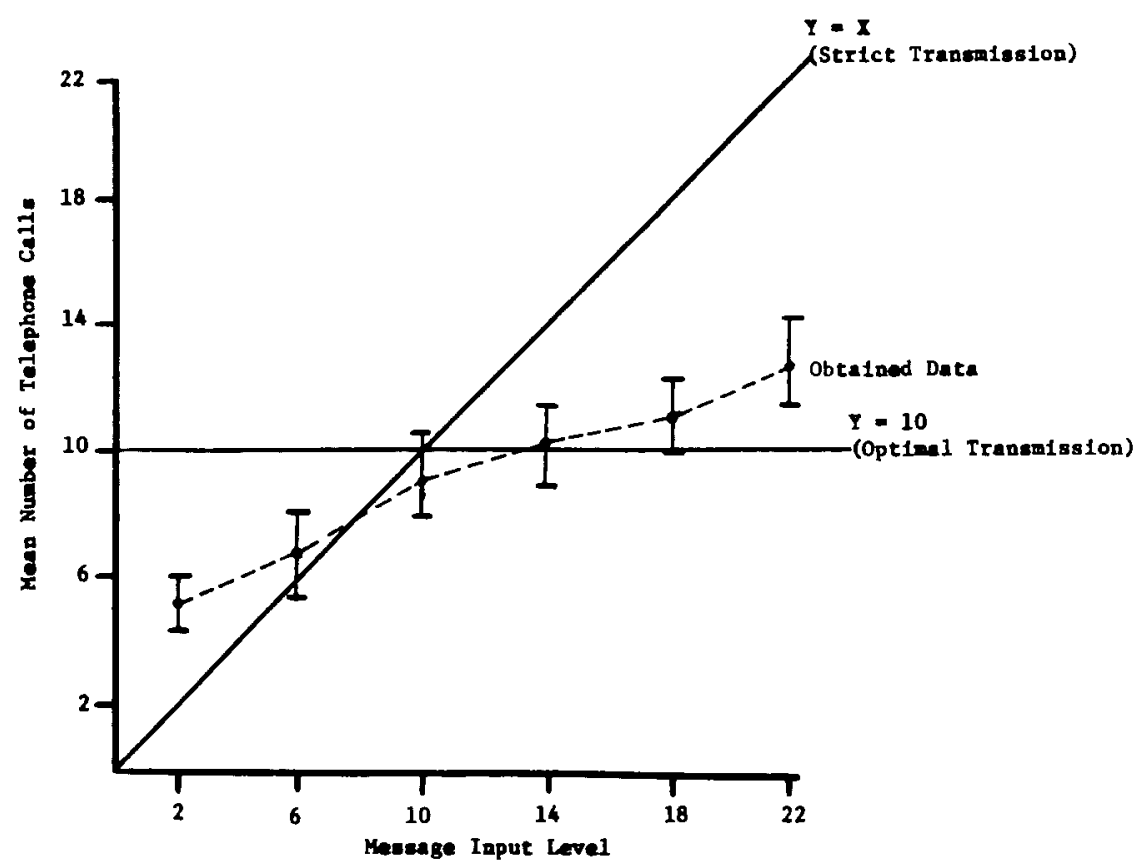

Fig. 1. Plot of obtained data with $95 \%$ confidence intervals and two predicted models (strict transmission and optimal transmission). 
possible predictive models are also plotted. One is a line derived from the potential assumption that human communication links produce a $1: 1$ input to output relationship (in which the number of calls made is predicted to equal the number of information messages received). The second line is obtained from the potential prediction that information acquisition groups would adjust information flow (telephone calls) to their decision-making counterparts, so that decision makers might engage in optimal complex decision making. This last prediction would suggest that the number of calls initiated by the information acquisition groups of each team should be constant at the level of 1 every $3 \mathrm{~min}$, or 10 every $30-\mathrm{min}$ playing period.

The data indicate that neither prediction held. Rather, the number of calls exceeded the number of information messages received by information acquisition groups when information load was low, but did not reach the level of optimality. Conversely, the number of calls slightly exceeded the optimal number under high information load levels, but remained well below the number of messages that were received. A check on the content of the calls (as recorded by the Ss) revealed that all calls made by all groups were relevant to the task at hand. Some of the information which information acquisition groups passed along to their decision-making counterparts was "imaginary" when information load was low, and the number of calls exceeded the number of informative messages that the $S s$ had received. Sixty-four percent of the information transmitted during Load Condition 2 and $17 \%$ during Load Condition 6 did not reflect any information that information acquisition groups had received (even though the content of the telephone messages was relevant to the group task). "Imaginary" information was not transmitted during Load Conditions 10, 14, 18, and 22 .

\section{DISCUSSION}

Even though no instructions were given to adjust the number of information calls to the decision-making groups of each team, the information acquisition groups did, in fact, increase the calls beyond those required by the available information when information was scarce and suboptimal, and decreased the calls when information load was superoptimal. If, as Campbell (1958) suggested, errors, particularly constant errors, serve an adaptive function, then one may propose that information acquisition groups reduced or increased their output to adjust their environmental constraints to their own liking. That adjustment would then serve to (even though it may be unintentionally) provide decision makers with a more optimal environment. If the previously reported research by Streufert and others holds, then the strategic (integrative) quality of the output from a decision-making group can likely be improved by introducing an intervening information acquisition (information gathering and sorting) group as a buffer between the information source(s) and decision-making groups.

A word of caution should be added. An improvement of decision-making quality by decision-making groups which is based on the adjusted information flow by information acquisition groups may or may not be a real improvement. If information acquisition groups add misleading information when information load is suboptimal (as they did in this research), and if they drop vitally important items of information when information is superoptimal, then the decision makers would base their (improved) strategic decision-making on faulty premises. Such decisions may be more strategic but may not achieve desired ends. Whether or not an adjustment of information flow via an information acquisition team is of value to a decision maker should probably be determined by considering the specific characteristics and potential outcomes of the decision maker's task.

\section{REFERENCES}

ALLPORT, G. W., \& POSTMAN, L. The psychology of rumor. New York: Holt, 1947 .
BARTLETT, F. C. Remembering, a study in experimental and sucial psuchology. London: Cambridge University Press. 1932 .

CAMPBELL. D. T. Systematic error on the part of human links in communication systems. Information \& Control, 1958, 1 334-369.

CAMPBELL, D. T., \& GRUEN, W Progression from simple to complex as a molar law of learning. Journal of General Psychology, 1958,59, 237-244.

DRIVER, M. J., \& STREUFERT, S. Group composition, input load, and group information processing. Institute for Research in the Behavioral, Economic, \& Management Sciences. Purdue University. Paper 142, 1966.

DRIVER, iM. J., \& STREUFERT $S$ Integrative complexity: An approach to individuals and groups as information-processing systems. Administrative Science Quarterly, 1969. $14,272-285$

SCHRODER, H. M., DRIVER, M. J.. \& STREUFERT, S. Iluman information processing. New York. Holt, Rinehart \& Winston, 1967 .

STREUFFRT, S. Complexity and complex decision making: Convergences between differentiation and integration approaches to the prediction of task performance. Journal of Experimental Social Psychology, 1970, 6, 494-509.

STREUFER'T, S.. CASTORE, C. H.. \& KLIGER, S. C. A tactical and negotiations game: Rationale, method and analysis. ONR Technical Report, Rutgers University, 1967.

STREUFERT, S. \& DRIVER, M. J Conceptual structure, information load and perceptual complexity. Psychonomic Science, $1965,3,249-250$.

STREUFERT, S., DRIVER, M. J., \& HAUN, K. H. Components of response rate in complex decision making. Journa of Experimental Social Psychology, 1967. 3, $236-\approx 95$.

STREUFERT, S., \& FROMKIN, H. L. Complexity and social influence. In $\mathrm{J}$. Tedeschi (Ed.), Social influence processes. Chicago: Aldine, 1972.

STREUFERT, S., KLIGER, S. C. CASTORE, C. H., \& DRIVER, M. J. A tactical and negotiations game for analysis of integration across decision areas. Psychological Reports, 1967, 20, 155-157

STREUFERT, S., \& SCHRODER, I1. M Conceptual structure, environmental complexity and task performance. Joumal of Experimental Research in Personality, 1965, 1, 132-137.

\section{NOTES}

1. Of course, neither decision makers nor information gathering-sorting groups know that optimal information load is equal to one item of information per $3 \mathrm{~min}$. However, past research of streufert and associates has repeatedly shown that Ss who can control information flow typically stabilize information search at that level.

2 . Includes $2 \mathrm{~min}$ out of $30 \mathrm{~min}$ lost by dialing a two-digit number. 